\title{
Overview of Wi-Fi Technology
}

\author{
Guo Yinan \\ School of Information and Electrical Engineering \\ China University of Mining and Technology \\ Xuzhou 221116, Jiangsu Province, China
}

\author{
Zhang Shuguo \\ School of Information and Electrical Engineering \\ China University of Mining and Technology \\ Xuzhou 221116, Jiangsu Province, China
}

\author{
Xiao Dawei \\ School of Information and Electrical Engineering \\ China University of Mining and Technology \\ Xuzhou 221116, Jiangsu Province, China
}

\begin{abstract}
Wi-Fi is a kind of new technology about wireless broadband access. Compared with conventional wireless technologies, it has faster speed, wider range and better security. In this paper, the concepts and characteristics of Wi$\mathrm{Fi}$ are introduced. Then the applications of Wi-Fi technology are given and several common construction methods of $\mathrm{Wi}-\mathrm{Fi}$ networks are presented. Problems existed in Wi-Fi technology are discussed. At last, the prospect of Wi-Fi's development is talked about.
\end{abstract}

Keywords-Wi-Fi, Ad-hoc, Infrastructure, Construction of the network

\section{INTRODUCTION}

With the widespread use of mobile terminals, such as laptop, Wireless Fidelity(Wi-Fi) phone, PDA, etc, the demand for wireless access has become increasingly prominent[1]. In recent years, the wireless networks developed rapidly. Among so many wireless standards, WiFi technology won the favor of people because of its lower construction and operating costs, higher data rate, farther transmission distance and better extensibility, etc.

$\mathrm{Wi}-\mathrm{Fi}$, so called wireless broadband, is widely applied in the mobile connection of home and small office network because of its flexibility and mobility. Especially in recent years, the number of wireless access point(AP) is increased rapidly. This makes the applications of wireless network more convenient and efficient. Besides, the APs can be set in public areas. Wireless metropolitan area networks exist in foreign countries based on wireless network standard. Some domestic universities also have been covered by the wireless local area networks(WLAN). Therefore, the position of WiFi in WLAN will become increasingly strong.

\section{OVERVIEW AND FEATURES OF WI-Fi TECHNOLOGY}

\section{A. Overview of Wi-Fi Technology}

According to its speed and the presented time, Wi-Fi technology can be divided into IEEE $802.11 \mathrm{~b}$, IEEE 802.11a, IEEE 802.11g and IEEE802.11n. IEEE802.11b and IEEE802.11g are commonly used [2]. IEEE802.11b is the oldest wireless network criterion and the most widely used Wi-Fi standard. Its maximum bandwidth is $11 \mathrm{Mbps}$. When the signal is weak or there are interferences, the bandwidth can be adjusted to $5.5 \mathrm{Mbps}, 2 \mathrm{Mbps}$ and $1 \mathrm{Mbps}$. The autoconditioning of bandwidth effectively ensure the stability and reliability of network.

IEEE 802.11a has a larger throughput than IEEE $802.11 \mathrm{~b}$. It works in the $5.8 \mathrm{GHz}$ frequency band and has good anti-interference ability. But it can not be compatible with IEEE $802.11 \mathrm{~b}$ and IEEE 802.11g. Moreover, its coverage is relatively small (only about $30 \mathrm{~m}$ indoor). So IEEE 802.11a is still rarely used among all the Wi-Fi standards currently.

In order to solve the incompatible problems between IEEE 802.11a and IEEE 802.11b, IEEE 802.11 working group formally approved the IEEE $802.11 \mathrm{~g}$ standard in July 2003 [2]. It can be compliant with IEEE 802.11 b. So the applications of IEEE 802.11g are more than IEEE 802.11a.

IEEE $802.11 \mathrm{n}$ standard approved in 2009 by IEEE is the latest Wi-Fi standard. It has a standard of $300 \mathrm{Mbps}$ and up to $600 \mathrm{Mbps}$ transmission speed [3]. The IEEE 802.11n standard combined MIMO and OFDM technology [4], not only to improve the quality of wireless transmission, but also greatly enhance the transmission speed. Comparison on Wi-Fi technology standard is shown in Table I.

TABLE I. COMPARISON OF WLAN STANDARDS

\begin{tabular}{cccc}
\hline items & frequency band & ton & modulation \\
\hline IEEE802.11 & $2.4 \mathrm{GHz}$ & $2 \mathrm{Mb} / \mathrm{s}$ & FHSS \\
IEEE802.11b & $2.4 \mathrm{GHz}$ & $11 \mathrm{Mb} / \mathrm{s}$ & DSSS \\
IEEE802.11a & $5.8 \mathrm{GHz}$ & $54 \mathrm{Mb} / \mathrm{s}$ & OFDM \\
IEEE802.11g & $2.4 \mathrm{GHz}$ & $54 \mathrm{Mb} / \mathrm{s}$ & DSSS \\
IEEE802.11n & $2.4 \mathrm{GHz} / 5.0 \mathrm{GHz}$ & $600 \mathrm{Mb} / \mathrm{s}$ & MIMO-OFDM \\
\hline
\end{tabular}

B. Features of Wi-Fi Technology

Wi-Fi is an effective way for broadband access. The features and advantages are mainly reflected in the following aspects [2-4]: 
(1)Long transmission distance. The radius of $802.11 \mathrm{n}$ standard is up to about $1000 \mathrm{~m}$.

(2)Fast transmission speed. Its transmission speed is very fast.The speed can reach $600 \mathrm{Mbps}$, which meets the personal and social needs.

(3)Compatibility with other services. In and above the second layer of Wi-Fi technology are fully consistent with the Ethernet.

(4)Convenient to form the network. Any devices with a wireless network adapter can be easy to enter the network. Therefore, it is very suitable for mobile requirement and has broad market.

(5)Security for use. The actual transmission power of IEEE 802.11 is only about $60-70 \mathrm{~mW}$. In contrast, the transmission power of cell phone is about $200 \mathrm{~mW}$ to $1 \mathrm{~W}$.The transmitter power of hand-held interphone is $5 \mathrm{~W}$. Therefore, Wi-Fi technology is absolutely safe.

\section{THE APPLICATIONS OF WI-FI TECHNOLOGY}

Since Wi-Fi technology was born at the end of the last century, it has developed for more than ten years. It was first used for indoor positioning by Bahl in 2000 [5]. With the popularization of mobile devices with $\mathrm{Wi}-\mathrm{Fi}$, its location technology is gradually extended from the interior to the outdoor[6]. In 2009, Nobuo Kawaguchi in Nagoya University introduced Wi-Fi-based positioning technology into subway information systems [7]. He also introduced the researches and applications of interior and outdoor Wi-Fi location technology[8]. In the Wi-Fi-based underground location and tracking systems, the communication between miner and manager and the positioning of the miners in complex environment have been solved [9]. So far, Wi-Fi location technology has widely used in the medical system. The physician can quickly get the patient's information and monitoring patients in time by Wi-Fi network [10].

Now Wi-Fi technology is integrated into more and more digital products. As the concept of digital home is proposed, a variety of products, such as televisions, DVD players, projectors, digital sound box, also have adopted Wi-Fi technology. These new types of appliances can be connected with the background media servers or computers via Wi-Fi network so as to realize digital and wireless of the whole family. As the concept of wireless city is proposed, many countries and regions have put forward the plans for Wi-Fi network coverage[11]. In 2008, a plan for "Wi-Fi through the Hong Kong Government" is present. In the United States, Houston government invested 3.5 million U.S. dollars to build the city's Wi-Fi network. Twelve cities in mainland China have made a clear plan for wireless cities. With the development of cities, Wi-Fi service in the future may be generally a public service and become a part of urban infrastructure construction.

\section{Wi-Fi NeTWORKING SCHEME}

\section{A. Wi-Fi Networking Equipments}

Wi-Fi is a wireless network consisting of wireless network adapter and AP [12-13]. AP is commonly called as access point or a network bridge, which is a bridge between traditional wired local networks and wireless local network. Wireless network adapter is the client device responsibling for receiving transmit signals from AP.

\section{B. The common Networking Scheme}

Compared with wired networks, the construction of wireless network is more flexible and convenient. For the networking with two computers, point-to-point structure can be used and the wireless $\mathrm{AP}$ is not required. For the networking with more than two computers, the infrastructure mode is used. A wireless AP (or wireless router) is adopted as the center of network [13].

(1)Ad-Hoc network

Ad-Hoc network is the simplest wireless LAN topology. It is also known as point-to-point network or peer-to-peer network. It consists of a group of computers with wireless interfaces (wireless client). These wireless clients share the same workgroup name, extended service set identifier(ESSID) and password. Any two sites on the network can communicate directly [2]. All nodes in selforganized network have the equal status[14]. Nodes can be joined and left the network at any time. The damage of node can not affect the entire network. So it has strong survivability. Ad-Hoc network topology is shown in Figure 1.

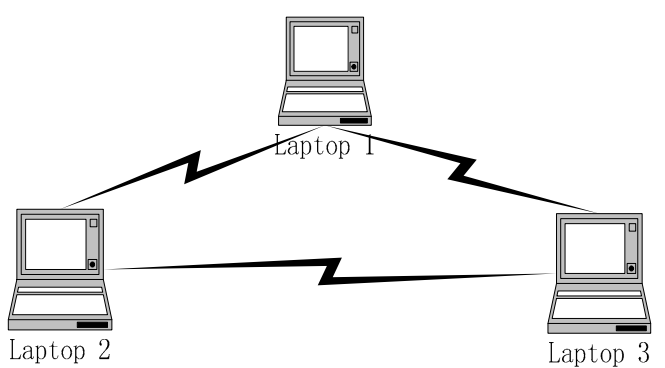

Figure1. Ad Hoc Wireless Network

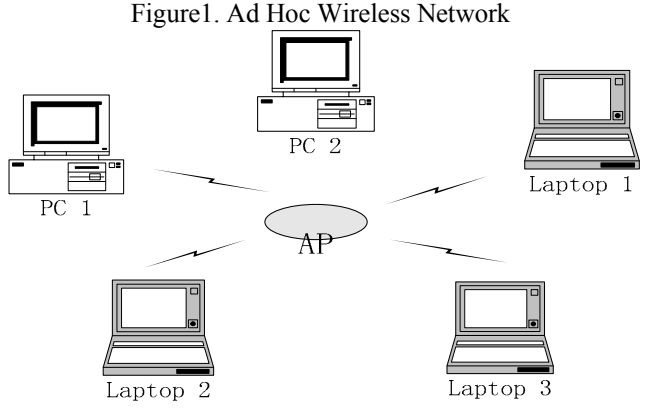

Figure2. Infrastructure Wireless Network

(2)Infrastructure Network

Infrastructure network is also known as the network with a center. It consists of one or more wireless AP and a series 
of wireless client[2]. The network topology is shown in Figure 2.

A wireless AP is used as a central station. All the wireless clients access to network by wireless AP. Since each site within the coverage of the central station can communicate with other sites, network layout confined by environment is relatively small. The largest shortage of infrastructure network is its poor survivability. The damage of central station is easy to result in paralysis of the entire network. Moreover, the central station increases the network cost.

\section{Outdoor Network Solution based on wireless bridge}

Wireless bridge is designed for interconnection between ad-hoc networks for transmiting long-distance data by wireless(microwave). It can be used to connect two or more separate network band. There are three main networking mode based on wireless bridge: point-to-point, point-tomultipoint, relay connection.

(1)Point-to-Point Model

Point-to-Point wireless bridge model generally consists of a pair of wireless bridge and a pair of outdoor antenna, shown as Figure 3. Directional antenna is often used as outdoor antenna. Directional antenna is helpful to improve the strength of wireless signals and the stability of wireless network. The mode is fit for the near local area networks and two wireless antennae can be seen each other.
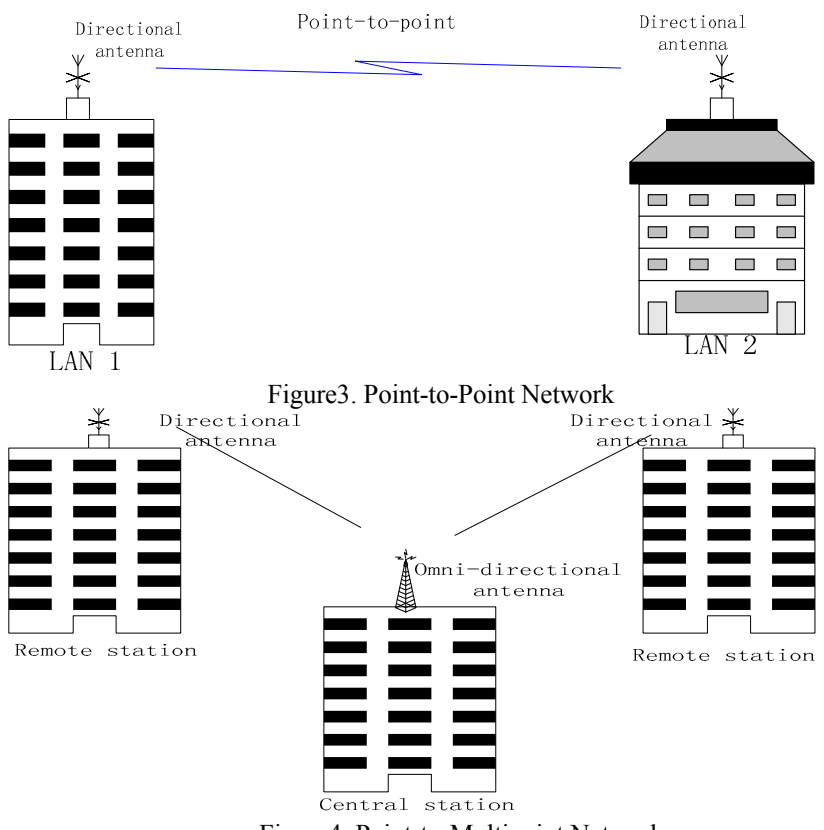

Figure4. Point-to-Multipoint Network

(2)Point-to-Multipoint Model

Point-to-Multipoint wireless bridge mode can combine a number of discrete LAN into one. This mode usually takes a network as the central station[15]. The other remote sites receive signals from the central station, as shown in Figure 4

In distributed cellular network, a sector manner is used to construct network. This also belongs to point-tomultipoint bridge mode. This mode is shown in Figure 5.

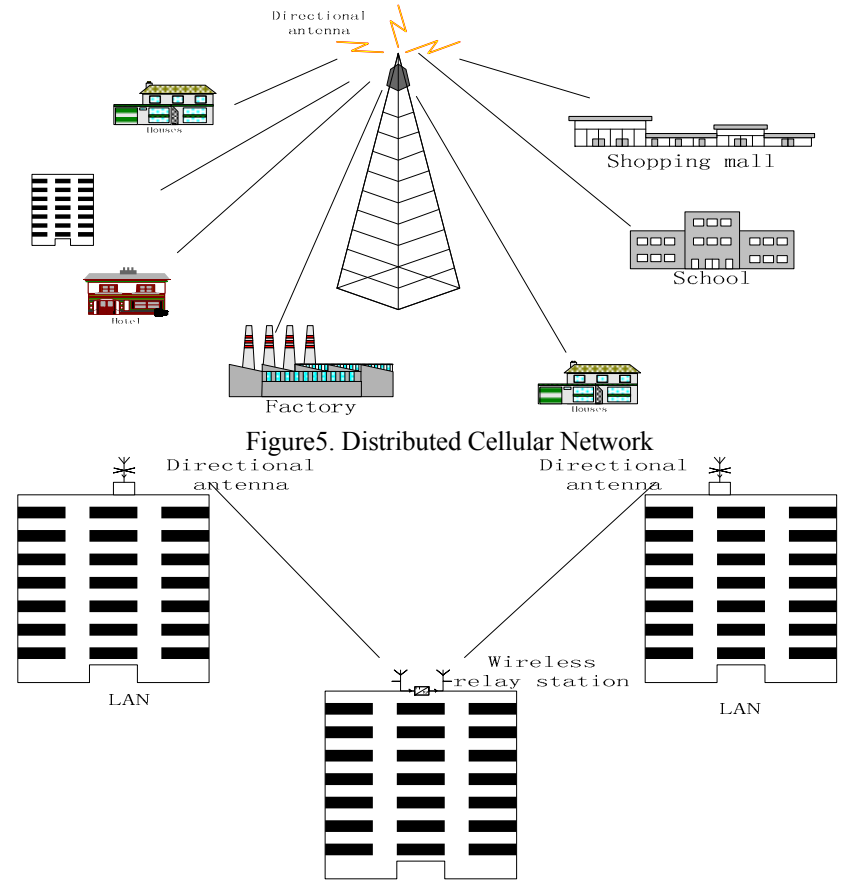

Figure6. Relay Network Model

(3) Relay Networking Model

Sometimes two LANs needed to be connected are blocked by the obstacles between them. At this time, wireless bridge can be used to bypass the obstacles so as to realize connection of the LANs, as shown in figure 6 . The location of the wireless relay stations must be able to see the other two directional antennae.

\section{PROBLEMS EXISTING IN WI-FI TECHNOLOGY}

The current Wi-Fi network is not perfect. There are many problems in the aspects of security, roaming switch and stability[16]. The current standards for Wi-Fi are not provided support for these problems.

\section{A. Security}

Because the transmission process of wireless signal is completely exposed to the air, it is more vulnerable than wired network to be hacked. If no proper security strategies, the network will face a larger risk. At the same time, "rub network" phenomena is very common in daily life. It seriously affects the speed and stability of the wireless network.

\section{B. Roaming Switch}

When we construct the wireless LAN, a large number of APs are deployed in order to cover larger-scale signals. When people move in the LAN, the terminals need to be switched among these AP.

The traditional switch idea used by Wi-Fi network is "first cut then connect". That is when the transition process is initiated, the workstation firstly cuts from the current AP, and then begins the normal switching process [16]. This 
may lead to the larger delay ( $>300 \mathrm{~ms})$, which affects the performance of mobile applications [17].

\section{Stability}

The wireless networks are easy to be disturbed by other signals around them due to the openness of the wireless channel. This results in the unsteadiness of Wi-Fi signals so as to effect on the quality of services. The main factors influenced on the stability of wireless network are: microwave oven $、 2.4 \mathrm{GHz}$ Cordless Phone and Mutual interference among the WLAN networks.

\section{SUMMARY AND OUTLOOK}

In this paper, the concept and characteristics of $\mathrm{Wi}-\mathrm{Fi}$ are briefly introduced. Then we summarize the applications of current Wi-Fi technology and present the use of Wi-Fi technology for indoor and outdoor networking solutions.

Roaming switching, security, interference, network stability for Wi-Fi technology are the problems shall be considered by network operators. Along with the development of internet protocol, Mobile IP technology and IPv6, roaming switching may be solved. In summary, the hotspot of Wi-Fi technology in future includes:

(1)Cellular Wi-Fi Network. We utilize the existing cellular radio communication network to connect numerous of WLANs in order to cover a larger area and build a "wireless city".

(2)Wi-Fi handsets and VoWLAN(Voice over WLAN) with high-definition sound quality services will become a potential application mode[18].

(3)Wi-Fi positioning technology. With the development of internet of things, Wi-Fi positioning technology[19] in real-time positioning and tracking of people and goods, environmental monitoring management will be more widely applied.

(4)Wi-Fi based wireless home network. The computers, televisions, printers, DVD players, digital projectors and other intelligent devices will be connected by Wi-Fi network to realize the digital and wireless of home appliances.

\section{ACKNOWLEDGMENT}

This work was supported by National Natural Science Foundation of China under Grant 60805025, National Natural Science Foundation of Jiangsu under Grant BK2010183 and Qinglan Project of Jiangsu.

\section{REFERENCES}

[1] Zhang Xinfeng. Research and Design of WiFi Function of the Embedded Multimedia Terminal [D].Shanghai: East China Normal University.2009

[2] Ma Xinluo; Li Xiaozhong; Ge Changtao . Construction and application of Wireless Local Area Network [M] . Beijing : National Defense Industry Press.2009

[3] Mei-Fang Lin, Jung-Yung Tzu, Lin, L., Huey-Ming Lee. The IEEE $802.11 \mathrm{n}$ capability analysis model based on mobile networking architecture[C]. in: Systems, Man and Cybernetics, 2009. SMC 2009. IEEE International Conference on,pp,1857-1860
[4] Nakamura, R., Sanada, Y. Experimental Investigation of IEEE802.11n Reception with Fractional Sampling[C]. in: Personal Indoor and Mobile Radio Communications (PIMRC), 2010 IEEE 21st International Symposium on,pp.1044-1048

[5] Bahl, P.,Padmanabhan,V.N..RADAR:An In-Building RF-based User Location and Tracking System[C]. Nineteenth Annual Joint Conference of the IEEE Computer and Communications Societies. Proceedings. IEEE,2000, (2):775-784

[6] Roberts, B.,Pahlavan, K.Site-Specific RSS Signature Modeling for WiFi Localization[C]. IEEE Conference of Global Telecommunications, 2009:1-6

[7] Kawaguchi, N.,Yano, M.,Ishida, S.,Sasaki, T.,Iwasaki, Y. ,Sugiki, K. Matsubara, S.Underground Positioning: Subway Information System Using WiFi Location Technology[C] . Tenth International Conference on Mobile Data Management: Systems, Services and Middleware,2009: 371-372

[8] Nobuo Kawaguchi,Furo-Cho, Chikusa-ku. WiFi Location Information System for Both Indoors and Outdoors, Lecture Notes in Computer Science [J], 2009:638-645

[9] Yongqiang Zhang,Liangliang Li ,Yongjian Zhang.Research and Design of Location Tracking System Used in Underground Mine Based on WiFi Technology[C]. International Forum on,Computer Science-Technology and Applications, 2010,(3):417-419

[10] Yanxia Liu,Liting Cao,Yuqiu Song. Application of WiFi Communication in Mobile Monitor [C].International Symposium on,Information Engineering and Electronic Commerce, 2009:313317

[11] Afanasyev, M.,Chen, T., Voelker, G. M.,Snoeren, A. C.Usage Patterns in an Urban WiFi Network, IEEE/ACM Transactions on Networking,[J], 2010:1-14

[12] Lyakhov, A.,Vishnevsky, V.. Unfair Access Problem in Wi-Fi Hot Spots[C]. IEEE 18th International Symposium on,Personal, Indoor and Mobile Radio Communications. 2007:1-5

[13] James F.Kurose Keith W.Ross.Computer Network[M].Beijing:Machinery Industry Press, 2005.

[14] Tian Zengguo,Liu Jingjing,Zhang Zhaoxian.Network Technology and Management[M]. Beijing: Tsinghua University Press,2009.

[15] WEN Xiao jun; WEN Guang bin. Project of Organizing Wireless LAN Based on the IEEE 802.11 Standards, Application Research of Computers [J],2002,(3):120-122.

[16] Gu Zhengyi. Wi-Fi-based wireless switching technology, Mobile Data $[\mathrm{J}], 2010,(1): 31-34$

[17] Yanfeng Zhang, Yongqiang Liu, Yong Xia. LeapFrog: Fast, Timely WiFi Handoff[C]. Washington, DC : Global Telecommunications Conference,2007: 5170 - 5174

[18] Mondal, A., Cheng Huang, Jin Li, Jain, M., Kuzmanovic, A. A Case for WiFi Relay: Improving VoIP Quality for WiFi Users[C]. Cape Town: Communications (ICC), 2010 IEEE International Conference on, 2010: 1-5

[19] Toyokazu Akiyama, Yuuichi Teranishi, Shingo Okamura Shinji Shimojo. A Consideration of the Precision Improvement in WiFi Positioning System[C]. International Conference on Complex, Intelligent and Software Intensive Systems:1112-1117 